\title{
El futuro de los sistemas de información en un régimen de gobierno abierto y ley de transparencia
}

The future of information systems in an open and transparent government environment

\author{
Emir SUAIDEN \\ Universidad de Brasília, Brasil, emir@unb.br
}

\begin{abstract}
Resumen
Los mayores desafíos de los países de América Latina para la implantación de la sociedad de información son la falta de un público lector, las deficiencias en la educación básica, la falta de una industria de contenidos y la precaria infraestructura informacional. Por esto, se calcula que poco más del veinte por ciento de la población latinoamericana puede ser considerada como incluida en la sociedad de la información. Para enfrentar este desafío es necesario que los sistemas informacionales adopten estrategias de inclusión productiva, o sea, estrategias que permitan preparar al ciudadano para el mercado de trabajo o para la generación de empleo y renta. En la nueva sociedad no es suficiente organizar el conocimiento: el desafío es transferir información y conocimiento.
\end{abstract}

Palabras clave: Inclusión digital. Latinoamérica. Políticas de información. Sociedad de la información. Sociedad del conocimiento. Alfabetización informacional.

\section{Contexto}

Para la realización de un análisis detallado del papel de la sociedad de la información en América Latina, debemos inicialmente describir la situación de la infraestructura social, educacional cultural e informacional existente en la región.

En el área social debemos destacar que los problemas aún existentes como la desnutrición infantil, el analfabetismo, el desempleo y la falta de mano de obra cualificada, generan en la práctica una mayor violencia urbana y rural. Una parte de la población no puede ser considerada incluida en el proceso de ciudadanía, pues desconocen sus derechos y deberes en la sociedad. Comprobadamente, el analfabeto es más dependiente y menos creativo; y automáticamente pasa a ser más fácilmente manipulado por la hegemonía existente. Los porcentajes de desempleo son cada vez mayores y verdaderamente muchos trabajadores no están capacitados para determinadas funciones. La juventud es la más afectada y muchas veces la alternativa es la huida hacía países desarrollados donde pasan a vivir en la

\begin{abstract}
The biggest challenges that Latin American countries face to become information societies are the lack of a reading public, the inefficiency of the educational system, the precarious state of the content industry and the deficient information infrastructure. Consequently, it is estimated that only about the twenty percent of the population can be considered included in the information society. To meet this challenge, information systems should implement productive inclusion strategies, that is, information inclusion strategies oriented to empower users for the labor market and employment and income generation. In the new information society, knowledge organization is not enough: the challenge is to transfer information and knowledge to the people that need them.
\end{abstract}

Keywords: Digital inclusion. Latin America. Information policies. Information society. Knowledge society. Information literacy.

clandestinidad. Cuando permanecen en su localidad muchas veces son seducidos por el narcotráfico.

Es imposible hablar de los problemas sociales sin abordar la cuestión educacional. El acceso a la educación aún es muy precario, principalmente en ciertas regiones de América Latina. Muchos alumnos frecuentan la escuela no por el conocimiento a adquirir, sino, fundamentalmente, por la merienda escolar, pues es la garantía de por lo menos una comida diaria. Además, existen profesores sin titulación que no tienen capacidad para difundir el proceso de enseñanza y aprendizaje. El nivel de repetición de cursos y absentismo es bastante alto. Del total de alumnos que ingresan en la escuela pocos llegan a la enseñanza superior.

En el ámbito cultural, la situación también es precaria. Los ministerios o secretarias de cultura existentes en América Latina son los que reciben, porcentualmente, los presupuestos más irrisorios. La información que circula en las comunidades marginadas aún es la información oral que, 
muchas veces, es obtenida en la iglesia, en la escuela y por los líderes locales. La cultura de la información en ciertas zonas de la región aún recuerda los tiempos de la colonización cuando el libro era para la elite dominante y la biblioteca un lugar de castigo.

Durante muchos años América Latina ha dado prioridad a la implantación de bibliotecas especializadas y centros de documentación, en detrimento de las bibliotecas infantiles, escolares y públicas. Ese hecho, añadido a la falta de conciencia de las autoridades y la inexistencia de una industria editorial fuerte, ha perjudicado la propagación de una política de diseminación de la lectura y de los hábitos de utilización de la información.

Aunque en los últimos años se ha comprobado el crecimiento de la industria de contenidos en la región, debemos destacar la dificultad que ciertas comunidades enfrentan para tener acceso al libro y eso compromete la implantación de una sociedad de la información. Generalmente, las grandes editoras están instaladas en los centros urbanos y presentan problemas de distribución en las zonas más necesitadas. Eso, unido al precio del libro -que es considerado inadecuado teniendo en cuenta el bajo poder adquisitivo de la comunidad-, contribuye al mantenimiento de esa situación. Hay países, en la región, donde el gobierno es el gran comprador, principalmente de libros didácticos y de textos. No obstante, los libros generalmente no llegan a las escuelas a inicio del semestre y muchas veces no son adoptados por los maestros.

Los estudios realizados en diversos países latino-americanos muestran que el acceso al libro depende del poder adquisitivo y de la formación educacional. La franja de la población que gana menos de cien dólares mensuales es muy grande, lo que impide la adquisición del libro y perjudica, obviamente, la formación del alumno. No obstante, debemos destacar que, en la región, la producción del libro de literatura infantil y juvenil ha crecido mucho y eso a largo plazo debe modificar la cultura de los alumnos que realizan sus investigaciones copiando diccionarios y enciclopedias. En las grandes ciudades, donde hay universidades de calidad y cursos de posgrado, el libro en formato electrónico está superando al libro impreso. Por lo tanto, América Latina enfrenta el desafío de pasar del impreso al digital.

No hay en la región una visibilidad de los problemas que afectan a la educación y la investigación, y eso se ve cada vez más claro en la medida en que la biblioteca escolar, la formación del hábito de la lectura y el uso de la información de la información no forman parte de los temas prioritarios de los dirigentes de las escuelas. Una revolución de ese modelo solamente tendría viabilidad si se realizara de afuera hacia dentro, o sea, de la comunidad asociada al resto del país, exigiendo formalmente la implantación de la biblioteca escolar con acervo actualizado, no solamente de libros didácticos, sino también de libros de literatura infantil y juvenil y principalmente con el profesional de la información actuando como mediador en el proceso enseñanza-aprendizaje.

En el actual contexto educacional de América Latina, el acceso a los medios de información no es tan importante como su comprensión. En ese sentido, principalmente para las poblaciones marginadas, comprender el valor de la información en el proceso de mejora de la calidad de vida pasa a ser la gran prioridad para la propagación de una política, basada y cimentada en la democratización y masificación de la información.

\section{Hacia la sociedad de la información}

Desde la década de los 90 los países considerados desarrollados intensificaron sus esfuerzos en la implantación de la sociedad de la información. En América Latina, con pocas excepciones, no existe aún una conciencia clara de este proceso. La sociedad de la información es el resultado de esas nuevas referencias sociales, económicas, tecnológicas y culturales, las cuales provocan un conjunto significativo de cambios en el ámbito de las sociedades y de sus organizaciones.

La información constituye la principal materiaprima, una fuente comparable a la energía que alimenta a un sistema; el conocimiento agrega valor a los productos y servicios; la tecnología pasa a ser un elemento vital para los cambios, en especial el empleo de la tecnología sobre acervos e información; la rapidez, la efectividad y la calidad constituyen factores decisivos de competitividad (Tarapanoff, 2001).

Se trata de una nueva sociedad que surge, con nueva estructura, nuevos canales de comunicación, nuevas formas de actuación social y de trabajo. Cambia la estructura del poder y de las instituciones, una nueva cultura y comportamiento se instalan, comprendidas y asimiladas, de forma más natural, completa, con mayor interés, y de forma intuitiva, por la nueva generación (Tapscott, 1997).

Esa nueva sociedad estaba prevista desde la década de 70. Lancaster (1977) anticipa la interacción global en el ámbito de la comunicación científica cuando afirma que el año 2000 sería el marco temporal de una sociedad sin papel. Según el referido autor, los científicos tendrían una 
vasta red de comunicación accedida a través de ordenadores que serían útiles no solamente para nuevos registros sino también para facilitar la producción científica. Afirmó también que las publicaciones científicas sufrirían un cambio radical, un proceso que habría de sustituir la tradicional forma de producción, distribución, y evaluación de los documentos, como ya se observa en la proliferación de los mecanismos de interoperabilidad en los archivos abiertos (LINCH, 2001). Por lo tanto Lancaster (1977) vislumbró una sociedad científica sin papel, donde los investigadores puedieran comunicarse de forma instantánea y globalmente, alterando, consecuentemente sus métodos de trabajo y la comunicación científica.

Así, es natural que esta revolución en los medios de la comunicación científica pase a exigir un nuevo profesional de la información, ya que en la nueva sociedad no es suficiente organizar el conocimiento y el desafío es transferir información y conocimiento.

El primer gran marco de interés de la implantación de la sociedad de la información en América Latina, fue la realización del seminario Sociedad de la Información en Mercosur y América Latina, realizado en Buenos Aires-Argentina del 22 al 24 de septiembre de 1999, y donde fueron sugeridas algunas directrices para el desarrollo del programa en el ámbito regional. En el ámbito de tecnologías de información se recomendaron políticas en tres áreas: infraestructura de la información y de la comunicación; contenidos de la sociedad de la información, y formación y estudios sobre las tecnologías de la sociedad de la información.

En lo que se refiere a los contenidos, se sugirieron las siguientes acciones: difusión de la información y estudios de impacto social de las nuevas tecnologías; fomento de sitios nacionales y regionales que incluyan los idiomas de Mercosur; promoción del desarrollo de sitios culturales, educacionales y de búsqueda; proposición de políticas e instrumentación de orientaciones para el uso terminológico, respetando los regionalismos; desarrollo de directrices para la selección de datos comunes; leLevantamiento de ítems comunes de meta datos para Mercosur; y desarrollo de instrumentos adaptados a la indexación con lenguaje controlado unificado (Seminario de especialistas, septiembre de 1999).

Tras el referido seminario, algunos países de la región, iniciaron tímidamente el debate sobre la implantación de la sociedad de la información. Brasil lanzó oficialmente, a través del Ministerio de Ciencia y Tecnología, el 15 de diciembre de
1999, el Programa Sociedad de la Información con la misión de:

- Articular, coordinar y fomentar el desarrollo y la utilización segura de servicios avanzados de computación, comunicación e información y de sus aplicaciones en la sociedad mediante la búsqueda, el desarrollo y la enseñanza, ofreciendo nuevos servicios y aplicaciones en Internet, y la garantía de ventaja competitiva y de la inserción de la empresa brasileña en el mercado internacional.

- Proporcionar ayudas para la definición de una estrategia destinada a estimular la inserción de la sociedad brasileña en la sociedad de la información. La obra Sociedad de la Información en Brasil: libro verde, en su versión oficial (septiembre de 2002) especifica las siguientes áreas de acción: mercado, trabajo y oportunidades; universalización de servicios y formación para la ciudadanía; educación para la sociedad de la información; contenidos e identidad cultural; gobierno al alcance de todos; tecnologías clave y aplicaciones; e infraestructura avanzada y nuevos servicios.

En lo que se refiere a contenidos y a identidad cultural, el Programa ofrece como líneas maestras la promoción de la generación de contenidos y aplicaciones que enfaticen la identidad cultural brasileña y las materias de relevancia local y regional; el fomento de esquemas de digitalización para la preservación artística, cultural, histórica y de informaciones en ciencia y tecnología; y los proyectos de investigación y desarrollo para la generación de tecnologías con aplicaciones en proyectos de relevancia cultural (Sociedad de Información en Brasil: libro verde, 2000).

Otra iniciativa importante, en el ámbito de América Latina, tuvo lugar en septiembre de 1998, Infolac-Programa Regional para el fortalecimiento de la Cooperación entre Redes y Sistemas Nacionales de Información para el desarrollo de América Latina y del Caribe, vinculado a la Unesco, que firmó la declaración de Panamá sobre la Sociedad del Conocimiento. Esta declaración formaliza la inquietud de los países miembros en relación con los diversos factores que influyen en la sociedad de la información, como la globalización de la economía de mercado, la expansión de la tecnología y su capacidad de acceso y uso, que imponen una política de contribución directa del sector de contenidos de información de los países en diversas actividades científicas, tecnológicas y económicas, con el objeto de estimular, en esos países, la creación de bases de información, el fortalecimiento de políticas públicas en el sentido de agilizar la competitividad global de la región y de garantizar el 
avance de la ciencia y de la competitividad tecnológica por ante la economía mundial. Las iniciativas en América Latina incluyen proyectos de desarrollo en Colombia, El Salvador, México, Argentina, Chile y otros (Ferreira y Tarapanoff, 1998/1999).

Debemos recordar que en las décadas de los 50 y 60 , organismos internacionales como la Unesco y a OEA, ante la falta de conciencia de los gobernantes de América Latina sobre la importancia de la Biblioteca Pública y Escolar, pasaron a invertir en el sector a través de la construcción de bibliotecas públicas, como la de Medellín, y a suministrar becas, capacitación y recursos financieros para el desarrollo de una política de información. No obstante, el objetivo mayor - que era la formación de un público lectorno fue alcanzado, pues no hubo una contrapartida adecuada de los gobernantes latino-americanos. Debemos considerar también las dificultades para implantar una cultura bibliográfica donde la información que circula es básicamente la información oral, principalmente en las clases de baja renta.

En un contexto social en el que se juntan sociedad de la información, la revolución tecnológica y la globalización, hay una seria ruptura en el sistema informacional, educacional y cultural. Ruptura de paradigmas aliada a una economía más competitiva, pues está basada en el conocimiento, provocando el surgimiento de nuevos valores que alcanzan al sistema educacional. Sintetizando los aspectos que derrumban los pilares del sistema escolar tradicional, podemos citar a Pérez Tomero (2000):

1. La escuela ya no es la depositaria privilegiada del saber, por lo menos no del saber socialmente relevante; es antes una fuente que compite además, con otras de enorme poder, tales como la televisión, la radio, los medios impresos y electrónicos, entre otros.

2. Las escuelas ya no son espacios privilegiados de transmisión de la educación, pues los medios de comunicación, o los grupos de iguales, se convierten en el más poderoso sistema educativo del momento.

3. La escuela es, tal vez, la institución más eficaz para la enseñanza de la lectura y de la escritura, pero se ha mostrado ineficiente en la promoción de la nueva alfabetización de la sociedad de la información.

4. En ese contexto, los profesores ya no son considerados los maestros que ostentan todas las habilidades y el conocimiento.

5. Las escuelas ya nos son las únicas instituciones que disponen de instrumentos para la producción, y la sistematización del saber. Sus bibliotecas, su tecnología, en general están desfasadas u obsoletas.

6. La escuela se ha alejado de la realidad. Situada entre el mantenimiento de un currículo tradicional y las exigencias de la nueva sociedad, encuentra serios problemas para hacer que sus enseñanzas sean útiles en la vida cotidiana.

7. La escuela está perdiendo la fuerza y el poder que el sistema social tradicional siempre le confirió. Esa realidad es la consecuencia del poco valor que esos poderes le atribuyen hoy. Si la formación escolar, por sí misma, no garantiza un empleo, la falta de formación es un factor decisivo para la exclusión. El problema se agrava especialmente en el caso de trabajadores con poca o ninguna calificación, y en el caso de los jóvenes que abandonan prematuramente la escuela. Para esos grupos de población, pueden ser insuperables las consecuencias de las innovaciones, principalmente de las tecnologías que modifican las estructuras y el funcionamiento de la economía y de la sociedad. Esa situación puede producir, en la región, divisiones tan profundas que lleven a la tensión y a la quiebra del tejido social, y generen continentes de excluidos e islas globalizadas (Seltzer, 2000).

\section{Hacia la sociedad del conocimiento}

En las formas de organización social que están surgiendo en la actualidad, la información y el conocimiento comienzan a tomar una dimensión, y a desempeñar un papel, que va más allá de lo que históricamente han cumplido. En las sociedades que desembocan en el tercer milenio, la posición de cada persona en el contexto social es, cada vez más, el producto del conocimiento que consiguió desarrollar o construir. La sociedad del mañana vislumbra una sociedad en la cual cada individuo y cada organización construirá su propia capacidad de acción, y por tanto, su posición en la sociedad, a través de un proceso de adquisición y desarrollo de conocimiento, y de la consolidación de su capacidad para generar nuevos conocimientos, que le permita adaptarse dinámicamente a una realidad en rápido y constante proceso de cambio y transformación.

Las sociedades que consiguen, a través de procesos continuos de generación y uso del conocimiento, organizados de tal forma que puedan contribuir al proceso de aprendizaje social, son clasificadas como Sociedad del Conocimiento. Estas últimas son sociedades con capacidad para generar conocimiento sobre su realidad y su 
entorno, y con capacidad para utilizarlo en su proceso de concebir, forjar y construir su futuro. Es decir, de utilizarlo en el proceso permanente de construcción de la sociedad, a través del desarrollo, transformación permanente, innovación y consolidación de sus propias instituciones sociales. De esta forma, el conocimiento se transforma no solo en instrumento para explicar y comprender la realidad, sino también en motor de desarrollo y factor dinamizador de los cambios sociales.

Por lo tanto, para hablar del papel de la sociedad de la información en América Latina, debemos destacar que en primer lugar debemos conocer la región que mezcla al mismo tiempo un alto potencial de riqueza unido a una población pobre. Pobre porque tiene un alto contingente de excluidos por la sociedad de la información, cuyos mayores indicadores son el analfabetismo, la desnutrición infantil y los desequilibrios en la distribución de la riqueza. En la propia región aún encontramos secuelas del tiempo de la colonización marcada aún hoy por el trabajo esclavo sin ninguna dignidad humana.

La reproducción del conocimiento es extremamente elitista, los más ricos tienen acceso a libros, bibliotecas y ordenadores de última generación, en cuanto los más pobres libran la batalla diaria por la supervivencia, lo que caracteriza la injusticia social. En un cuadro caracterizado por las desigualdades sociales las preguntas más frecuentes son:

1. ¿Es posible acabar con las desigualdades sociales en América Latina, basándose en la concepción de la sociedad de la información?

2. ¿Cómo incluir en la sociedad de la información comunidades sin poder adquisitivo y sin oportunidades de acceso al sistema educacional y al mercado de trabajo?

3. ¿Cuál sería la función de los profesionales de la información en una sociedad de la información teóricamente más justa?

Para responder a las citadas preguntas, debemos destacar que solamente un cambio radical en los planes de formación de recursos humanos en el área de la ciencia de la información podría fomentar una revolución social para acabar con la brecha, que posibilitase a la gran comunidad latinoamericana visualizar los caminos hacia la inclusión en la sociedad de información, un cambio en los currículos de las escuelas de biblioteconomías y ciencia de la información tanto a nivel de bachillerato como de posgrado, que posibilite la creación de disciplinas y líneas de investigación sobre la dimensión humana de la informa- ción, o sea alinear la teoría a la practica en el proceso de difundir informaciones que sean relevantes para el crecimiento educacional de las comunidades de baja renta, y también la creación de disciplinas en investigación sobre la mediación de la información.

La dimensión humana de la información tendrá como foco principal el establecimiento de diagnósticos adecuados sobre la circulación de la información en los núcleos de población de baja renta.

- ¿Cuál es la cultura de la información? ¿Qué tipo de información circula?

- ¿Cuál es la estructura educacional, social y cultural de la comunidad? ¿Cuáles los productos y servicios más adecuados para aquél tipo específico de comunidad?

- ¿Cómo sería la interacción de los servicios de información con la comunidad? ¿Habrá necesidad de segmentar el mercado?

- ¿Cuáles son los indicadores de impacto en la utilización de los servicios de información?

Todas esas preguntas serán respondidas por la disciplina dimensión humana de la información que se utilizará como técnica para toma de decisión, inteligencia organizacional y capital cultural para dar una dimensión científica a las enseñanzas.

Otro tema relevante y de incuestionable valor para la inclusión en la nueva sociedad es la mediación de la información. Sabemos que en América Latina el acceso a los libros y a los servicios de información es aun extremamente elitista. Con el advenimiento de la revolución tecnológica ese gran contingente de excluidos pasó, en algunas regiones, a tener acceso a las nuevas tecnologías. Se pasó así de la desinformación al exceso de información o la llamada explosión de información. El clima creado es de frustración e impotencia ante el nuevo mundo y las preguntas más frecuentes son: qué seleccionar, cómo seleccionar, para qué seleccionar, cuál es la actualidad de la información, cuál la oposición al discurso, qué dicen las entrelíneas y cuál la importancia de esa búsqueda y de la calidad de los textos en un escenario de selección para la selectividad y para el mercado de trabajo.

Se crea un clima adecuado para la alfabetización en información que precede metodológicamente a la alfabetización digital. En la alfabetización en información lo básico es aprender a aprender y donde se trabaja con habilidades individuales. En una sociedad intensiva en información, en que el ciudadano interactúa con personas y máquinas 
en un constante intercambio de datos y de información, la alfabetización tradicional, las habilidades de lectura y de escritura que constituyen la base de los sistemas educativos primarios no son suficientes. A esas habilidades habrá que agregarles otras, tales como el saber navegar por fuentes infinitas de información; el saber utilizar los sistemas de información, el saber identificar la calidad de la fuente, el saber determinar su confiabilidad, el saber dominar la sobrecarga de información; o saber aplicar la información en problemas reales, el saber comunicarle a otros la información encontrada, y, por encima de todo: el saber utilizar el tiempo - el verdadero recurso escaso en la sociedad del conocimiento- para aprender constantemente (Cornellá, 1999).

Por lo tanto creemos que los dos caminos propuestos: valorización de la dimensión humana de la información y mediación de la información serán vitales para la inclusión de la población marginal de América Latina en la sociedad de la información. La primera - dimensión humana de la información- demuestra que la información no es solamente un bien económico, un bien de la hegemonía o de los representantes del poder, sino, fundamentalmente, un bien social donde la figura más importante es el ser humano que pasa ser valorizado y a tener dignidad en la medida en que la información con valor agregado significa conocimiento y ese conocimiento posibilita el acceso al empleo y a la mejora de la calidad de vida.

La segunda -mediación de la información- posibilita el acceso a las fuentes de la información utilizando metodologías de alfabetización de la información, que posibilitan la creación de indicadores de impacto social y de indicadores en el proceso enseñanza-aprendizaje, que disminuyen el fracaso escolar —básicamente medido en América Latinapor la repetición de cursos y el absentismo escolar-. En ese proceso el mediador utiliza información bibliográfica y digital para dar mayor calidad a la investigación y principalmente en la formación de ese nuevo ciudadano que debe ser incluido en la sociedad de la información.

El primer paso del mediador es la elaboración del diagnóstico de las necesidades de información de su comunidad. Generalmente en América Latina, las comunidades más carentes tienen una necesidad mayor de tener acceso a las informaciones útiles, también llamadas informaciones solidarias o informaciones para la ciudadanía. Esas informaciones son utilizadas para la resolución de los problemas cotidianos, y su disponibilidad y difusión son procesos complejos, pues muchas veces la situación educacional del futuro usuario no permite la lectura y comprensión de textos. La transmisión de ese tipo de información, en la mayoría de las veces, es a través de la información oral. El segundo paso es la conducción de esa comunidad a la información bibliográfica, que exige lectura y capacidad crítica en la interpretación de los textos. El paso siguiente es la información digital que, principalmente en América Latina, produce una motivación mayor en la comunidad que la información bibliográfica. A continuación y siempre utilizando técnicas de toma de decisión y metodologías adecuadas al proceso, el mediador crea indicadores de impacto social que sirven de evaluación en el proceso de transferencia de la información.

En una planificación estratégica esos procedimientos son esenciales para acabar con las barreras que impiden el desarrollo de América Latina en el proceso de poner fin a las desigualdades reinantes en el área del acceso y comprensión de la información.

\section{Conclusiones}

Las transformaciones tecnológicas operadas en el campo de las telecomunicaciones y la computación han provocado cambios en la sociedad, han cambiado las formas de trabajo y las formas de interacción y comunicación de grandes sectores de la sociedad como el científico, el económico, el social, el educacional, el de la información, entre otros.

En un régimen de gobierno abierto y ley de transparencia cabe a los sistemas de información facilitar el acceso libre a las fuentes permitiendo por medio de un trabajo ético la comprovacion de las informacionales en todos los sentidos.

En la actualidad las nuevas formas de difusión ponen al alcance de estos sectores el acceso a un universo de información nunca antes imaginado, sin límites de fronteras, a altas velocidades y con una cobertura universal que satisface las más sofisticadas necesidades de un usuario en cualquier punto geográfico del planeta, con el requerimiento fundamental de contar con una computadora y una conexión a la red de redes. En este contexto es fundamental que el usuario tenga la competencia informacional para el disfrute de este nuevo escenario.

Ante este panorama, concertado bajo las implicaciones sociales del progreso tecnológico, la información constituye el ingrediente clave a partir de cual la sociedad participa en procesos de cohesión, globalización, informatización y generación del conocimiento; mientras que los bibliotecarios, especialistas en información, bibliotecas y otras unidades de información son los in- 
termediarios que, ante la sociedad, juegan el papel protagónico en la selección, procesamiento y difusión de esta información, considerada como un relevante recurso para el desarrollo social.

Esta realidad, concebida por especialistas como el cambio social más importante operado desde la revolución de Gutenberg hasta nuestros días, se ha convertido en objeto y tema de estudios de muy diversos especialistas provenientes, en lo fundamental, del campo de las ciencias sociales, quienes han coincidido en señalar que este cambio ha provocado el origen de nuevos paradigmas en los que se acentúa un proceso de transformación de las anteriores relaciones sociales y que deja entrever en el horizonte el surgimiento de una nueva forma de organización social, a la que todos coinciden en bautizar con el nombre de sociedad de la información.

La revolución tecnológica, la globalización y el adviento de la sociedad de la información fueron factores fundamentales y demostraron que el profesional de la información puede actuar como factor de cambio para la construcción de una nueva sociedad con más justicia social. Para esto, tiene que tener una formación más adecuada y compatible con los desafíos de esta sociedad. El primero paso es trabajar como mediador de la información, sea esa información bibliográfica, digital o virtual. El profesional con esa metodología puede acabar con la cultura, en América Latina, de copiar los textos y pasar a trabajar con la autoría. O sea el usuario deja de ser dependiente de la información y se transforma en productor de la información. Con esto se aumenta en cada país la producción científica y la generación de patentes. El país pasa a ser menos dependiente y puede construir su propio futuro.

Sabemos que muchas bibliotecas adormecidas cuentan en general con fondos y tecnologías adecuadas. No obstante, muchas veces están repletas de usuarios que practican la copia de diccionarios y enciclopedias. Hay que formar un nuevo tipo de usuario que pueda producir investigación de calidad.

Con el adviento de la revolución tecnológica, por un lado las personas están más conectadas, por otro están más impersonales y distantes. Por lotanto es impresindible que las bibliotecas capaci- ten sus usuarios para transformarlos en productores de información, o sea, saliendo de la copia hacia la autoria.

Muchos sistemas de información ya desarrollan programas de inclusión productiva que pueden ser considerados como buenas prácticas. El Servicio Brasileño de Respuestas Técnicas (SBRT) reúne informaciones tecnológicas para proporcionar al pequeño y medio empresario condiciones para el éxito de sus negocios. Muchas bibliotecas públicas ya segmentan los productos y servicios para proporcionar empleabilidad a sus comunidades. En las zonas suburbanas e rurales, el éxito de los agronegocios se debe al suministro en tiempo real de información adecuada para el aumento, producción e distribución de los alimentos.

\section{Referencias}

Cornella, A. (1999). La education en la sociedad del conocimiento. Extranet, mensaje 419. http/www.extra-net.net.

Ferreira, J. R.; Tarapanoff, K. (1998/1999). Sociedade da informação: conteúdosinformacionais; proposta do IBICT. // Informado; Revista de la Escuela Universitária deBibliotecologia y Ciências Afines. 3/4, 147-162.

Lancaster. F. W. (1977). The dissemination of scientific and tecnical information: toward a paperless system. Urbana III, University of Illinois. 1977.

Lynch, Clyfford (2001). Metadada: haversting and the open archives initiative. ARL Bimonthly Report n.217, aug.2001. www.arl.org/newsltr/217/PMH.html.

Oliveira, C. L. (2003). A revolução tecnológica e a dimensão humana da informação: a construção de um modelo de mediação. Brasília, Universidade de Brasília. (Tese dedoutorado aprovada pelo departamento de Ciência da Informação e Documentação daUniversidade de Brasília).

Pérez Tornero, J. M. (2000). Comunicación y education en la sociedad de la information: nuevos lenguajes y consciência crítica. Barcelona: Gedisa.

Seltzer, K.; Bentley, T. (2000). La era de la creatividad: conocimientos y habilidadespara una nueva sociedad. Madrid: Santillana.

Takahashi, T. (org.) (2000). Sociedade da informação no Brasil: livro verde. Brasilia: Ministério da Ciência e Tecnologia.

Tapscott, D. (1997). Growing up digital: the rise of the net generation. New York: McGraw Hill.

Tarapanoff, K. (2001). Inteligência organizational e competitiva. Brasilia: Universidade de Brasilia.

Enviado: 2016-06-28

Aceptado: 2016-09-20. 
